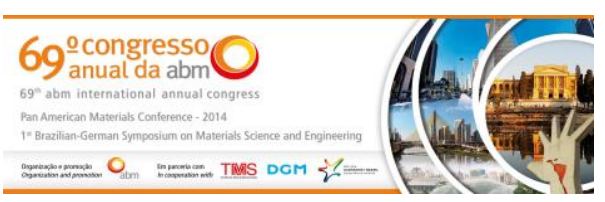

Tema: Diagramas de fases e transformações de fases

\title{
INFLUÊNCIA DA COMPOSIÇÃO QUÍMICA NA LINGOTABILIDADE DE AÇOS*
}

\author{
Roney Eduardo Lino' \\ Ângelo Máximo Fernandes Marins ${ }^{2}$ \\ Leandro Aparecido March/ ${ }^{3}$ \\ Jamylle Assis Mendes ${ }^{4}$ \\ Lucas Vieira Penna ${ }^{5}$ \\ Joaquim Gonçalves Costa Neto ${ }^{6}$ \\ João Henrique Palmer Caldeira ${ }^{7}$ \\ André Luiz Vasconcellos da Costa e Silva ${ }^{8}$
}

\section{Resumo}

Melhorar a qualidade dos aços e dos processos tem sido uma rotina dos engenheiros metalurgistas e das siderúrgicas em um mercado que exige produtos com qualidade e preço competitivos. No processo de refino dos aços são feitos os ajustes de composição química e temperatura, além das modificações inclusionárias necessárias às características de qualidade dos produtos e as exigências para uma perfeita lingotabilidade. O processo de solidificação mais comum é o lingotamento contínuo, onde a continuidade de fluxo de aço líquido através das válvulas é assegurada através da temperatura adequada e presença de inclusões no estado líquido nas temperaturas de lingotamento. Neste trabalho foram utilizados os softwares Thermocalc e CEQCSI com o objetivo de avaliar o efeito dos elementos químicos carbono, silício e enxofre na janela de lingotabilidade de diagramas de fases alumínio vs cálcio. Os softwares se mostraram bastante apropriados e eficazes neste estudo, e os resultados indicam que os elementos estudados afetam diretamente a posição da janela de lingotabilidade dos aços carbono. A análise de amostra de tarugo de um aço $0,2 \% \mathrm{C}$ via MEV mostrou que há grande heterogenidade de inclusões no aço acalmado ao alumínio e tratado com cálcio.

Palavras-chave: Diagrama de fases; Thermocalc; Lingotamento contínuo; Cálcio.

\footnotetext{
INFLUENCE OF CHEMICAL COMPOSITION ON STEEL CASTING PERFORMANCE Abstract

Improving the quality of steel and its manufacturing processes have always been a matter of routine for metallurgical engineers and steelmakers, this being a requirement in a market that demands quality products at a competitive price. Adjustments in chemical composition and temperature are performed in the secondary metallurgy refining process, as well as the necessary inclusion modification. The most common solidification process is continuous casting, where the continuous flow of molten steel through valves is maintained through controlled temperature and the presence of inclusions in the liquid state at casting temperatures. In this study, Thermocalc and CEQCSI softwares were used with the aim of evaluating the effect of the chemical elements carbon, silicon and sulfur on the castability window in aluminum vs calcium phase diagrams. The softwares proved very appropriate and effective in this study, and the results indicate that the elements studied directly affect the position of the castability window for carbon steels. The analyze of a billet sample $0.2 \% \mathrm{C}$ by SEM show a large heterogeneity of inclusions in a aluminum killed and calcium treated steel.

Keywords: Phase diagram; Thermocalc; Continuous casting; Calcium.

Eng. Metalurgista, ArcelorMittal Monlevade, MSc, Prof., FAENGE, UEMG, João Monlevade, MG, Brasil. Eng. Metalurgista, MSc., Eng. de produção, Aciaria da ArcelorMittal Monlevade, João Monlevade, MG, Brasil. Eng. Mecânico, ArcelorMittal Monlevade, MSc, Prof. da FAENGE, UEMG, João Monlevade, MG, Brasil Estudante, Engenharia Metalúrgica, FAENGE, UEMG, João Monlevade, Minas Gerais, Brasil.

Eng. Metalurgista, MSc, Gerente de Aciaria, ArcelorMittal Monlevade, João Monlevade, MG, Brasil.

Eng. Metalurgista, Coord. Assistência Técnica, ArcelorMittal Monlevade, João Monlevade, MG, Brasil.

Eng. Metalurgista, Diretor Industrial, ArcelorMittal Monlevade, João Monlevade, MG, Brasil.

Eng. Metalurgista. PhD, Diretor Técnico do IBQN, Professor da EEIMVR, UFF, Volta Redonda, RJ, Brasil.
}

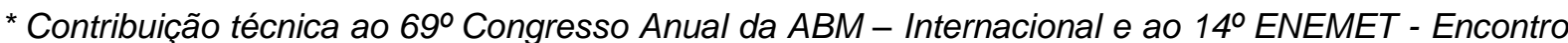
Nacional de Estudantes de Engenharia Metalúrgica, de Materiais e de Minas,21 a 25 de julho de 2014, São Paulo, SP, Brasil.
} 


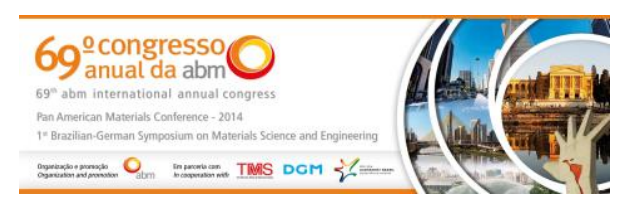

\section{INTRODUÇÃO}

Para se produzir tarugos de aço via lingotamento contínuo é necessário o correto tratamento do aço na panela para o controle adequado dos produtos da desoxidação e demais inclusões [1,2]. A presença de inclusões líquidas após o refino secundário é considerada um pré-requisito para o fluxo sustentável de metal através dos pequenos orifícios das válvulas do lingotamento. A lingotabilidade do aço é influenciada principalmente pela presença de microinclusões sólidas no aço líquido, sendo a taxa de deposição de inclusões nas válvulas uma função da concentração dessas inclusões no aço líquido [3].

O ajuste de composição química no forno-panela pode ser feito com bastante precisão, assim como a dessulfuração e o tratamento do aço com cálcio. A adição de cálcio em fio permite alterar o estado físico das inclusões geradas no processo de desoxidação. A passagem de sólido para líquido é, evidentemente, função da temperatura liquidus destas partículas. As fases presentes nas inclusões em equilíbrio em uma temperatura específica podem ser estimadas através de diagramas de fase cálcio vs alumínio, onde a fase líquida define a região dos diagramas denominada "janela de lingotabilidade" [4-6].

Além de permitir o fluxo ininterrupto de aço no processo lingotamento contínuo, a modificação da morfologia das inclusões através do tratamento com cálcio proporciona um importante incremento na qualidade de aços ligados de alta resistência. A propriedade de resistência à fadiga requerida em determinadas aplicações depende diretamente da composição química, forma e distribuição de tamanho das inclusões, por exemplo. Inclusões angulares, longas e duras devem ser evitadas, pois elas não podem ser deformadas junto com a matriz de aço durante a laminação a quente, o que pode resultar em uma concentração de tensões na interface aço/inclusão [7]. A Figura 1 mostra, de forma esquemática, como a morfologia das inclusões pode ser modificada com o tratamento com cálcio [8]. Neste esquema, as inclusões em um aço desoxidado ao alumínio e tratado com cálcio são inclusões esféricas com o centro a base de aluminato de cálcio de baixo ponto de fusão, coberto por sulfetos de cálcio e manganês. Inclusões com ponto de fusão relativamente mais baixo, menores em tamanho e com morfologia esférica são mais indicadas para a melhoria das propriedades de resistência a fadiga e da tenacidade dos aços e para evitar ou reduzir a fragilização por hidrogênio [1,7,9-11].

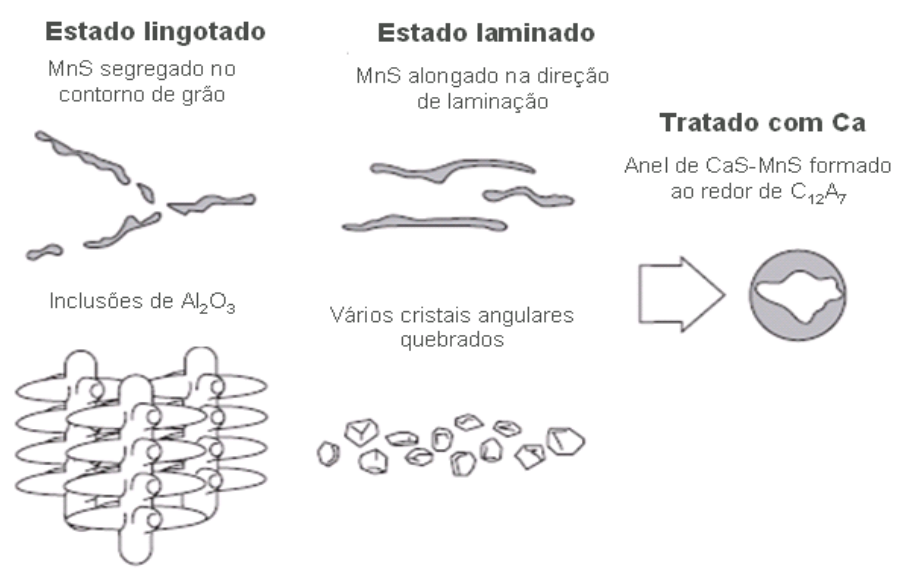

Figura 1. llustração esquemática da modificação da morfologia da inclusão em função do tratamento com cálcio [8].

\footnotetext{
* Contribuição técnica ao $69^{\circ}$ Congresso Anual da ABM - Internacional e ao 14ํㅡㄴ ENEMET - Encontro Nacional de Estudantes de Engenharia Metalúrgica, de Materiais e de Minas,21 a 25 de julho de 2014, São Paulo, SP, Brasil.
} 


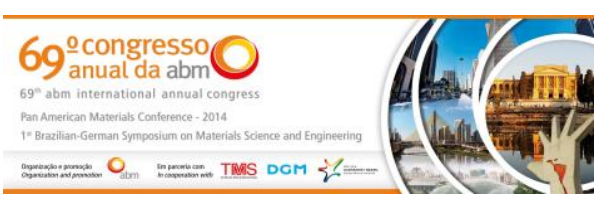

O presente trabalho tem o objetivo avaliar a influência do nível de enxofre e dos elementos desoxidantes silício e carbono nas janelas de lingotabilidade de aços produzidos na rota forno-panela $\rightarrow$ lingotamento contínuo.

\section{MATERIAIS E MÉTODOS}

Foram selecionadas 54 composições químicas de aços variando os percentuais dos elementos carbono $(0,2 \%, 0,4 \%$ e $0,6 \%)$, silício $(0,02 \%$ e $0,20 \%)$ e enxofre $(0,005 \%$, $0,010 \%$ e 0,015\%), equivalentes a ligas comercialmente produzidas (ex: SAE 1020, SAE 1040 e SAE 1060). Para essas composições químicas de aços foram calculadas as temperaturas liquidus utilizando 0 software de termodinâmica computacional Thermocalc, uma vez que este software é capaz de estimar com boa precisão a temperatura liquidus para uma ampla faixa de composição química [12].

Os diagramas de fase alumínio versus cálcio foram calculados para as 54 ligas empregando o software Thermocalc e o banco de dados SLAG2. Os diagramas foram calculados em função dos teores de alumínio e cálcio a uma temperatura $50^{\circ} \mathrm{C}$ acima da temperatura liquidus da liga. Apesar de poderem afetar a "janela de lingotabilidade", o teor de oxigênio total e a pressão utilizados nas simulações foram mantidos fixos em 20ppm e 1atm respectivamente, e os demais elementos presentes no aço foram, também, mantidos constantes. Os efeitos desses fatores serão avaliados em trabalhos futuros.

A partir destes diagramas alumínio versus cálcio foram identificados os teores mínimos e máximos de cálcio necessários para se obter inclusões líquidas em função do teor de alumínio, sendo o valor visado igual a média entre os valores mínimos e máximos. Utilizando os valores visados de cálcio obtidos na simulação e a composição química das ligas foi feita uma regressão múltipla utilizando o software Minitab.

Foram analisadas amostras de tarugos lingotados de alguns aços produzidos industrialmente via lingotamento contínuo. As inclusões foram analisadas através de Microscópio Eletrônico de Varredura (MEV), onde os tipos de óxidos presentes nas mesmas foram identificados em análise semi quantitativa. A temperatura liquidus e a fração de fase liquida das inclusões foram calculadas em função da composição química através do software termodinâmico CEQCSI (módulo Cristal).

A Figura 2 mostra a tela principal dos softwares termodinâmicos utilizados na ArcelorMittal Monlevade.

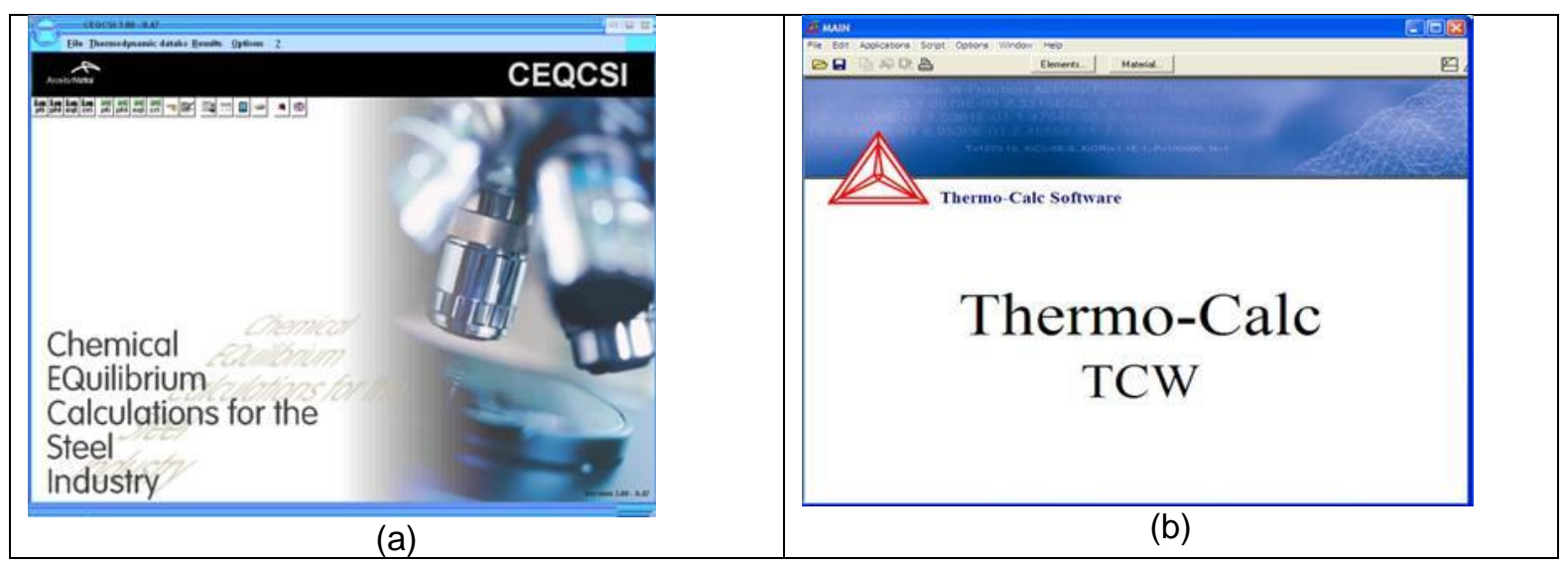

Figura 2. Telas dos softwares termodinâmicos a) CEQCSI e b) Thermocalc

\footnotetext{
* Contribuição técnica ao 69 Congresso Anual da ABM - Internacional e ao $14^{\circ}$ ENEMET - Encontro Nacional de Estudantes de Engenharia Metalúrgica, de Materiais e de Minas,21 a 25 de julho de 2014, São Paulo, SP, Brasil.
} 


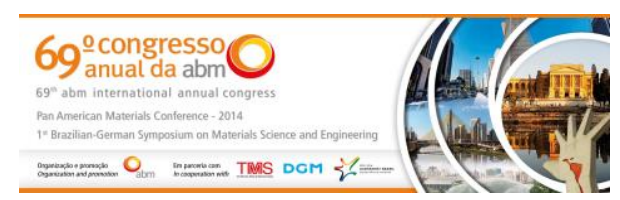

\section{RESULTADOS E DISCUSSÃO}

Para todas as composições químicas propostas foram calculados os diagramas equilíbrio de fases alumínio-cálcio a temperatura $50^{\circ} \mathrm{C}$ acima da temperatura liquidus das ligas, mantendo os demais elementos constantes, como descrito acima. Como é sabido, o teor de carbono influencia diretamente a temperatura de fusão da liga, passando de $1521^{\circ} \mathrm{C}$ em um aço baixo teor de carbono $(0,2 \% \mathrm{C})$ para $1486^{\circ} \mathrm{C}$ em um aço alto teor de carbono, o que pode ser observado na Tabela 1. O conhecimento da temperatura liquidus e o controle da temperatura do aço nos processos de refino e lingotamento é de fundamental importância para garantia da continuidade do processo produtivo da aciaria [7].

Tabela 1. Temperatura liquidus média das ligas em função do teor de carbono.

\begin{tabular}{c|c}
\hline Carbono da liga & Temperatura liquidus média calculada \\
\hline $0,2 \%$ & $1521^{\circ} \mathrm{C}$ \\
\hline $0,4 \%$ & $1504^{\circ} \mathrm{C}$ \\
\hline $0,6 \%$ & $1486^{\circ} \mathrm{C}$ \\
\hline
\end{tabular}

A Figura 3a mostra os diagramas alumínio vs cálcio para um aço com $0,2 \% \mathrm{C}$, $0,05 \%$ S e $0,02 \%$ Si (e 20ppm de Ot). Na figura estão indicadas fases sólidas de aluminatos de cálcio, de silicatos de cálcio e sulfetos, assim como a presença de inclusões liquidas (SLAG) na temperatura da simulação. A área identificada no diagrama para essas inclusões líquidas é de grande importância industrial, uma vez que representa a região da "janela de lingotabilidade" [4]. Essa área, que representa uma faixa específica de teor de cálcio, deve ser buscada para se garantir uma boa lingotabilidade do aço. $O$ fato das inclusões desta região estarem no estado líquido favorece a forma esférica que é muito mais fácil de ser removida do aço [7]. Acima dessa área à esquerda (baixo alumínio e alto cálcio) é identificada a fase sólida de silicato de cálcio. À direita dessa região (alto alumínio e alto cálcio) aparecem as fases de sulfeto de cálcio juntamente com inclusão liquida. Abaixo da janela de lingotabilidade está a região de aluminato de cálcio sólido com inclusões liquidas e logo abaixo as região de inclusões sólidas de aluminatos de cálcio. Todas as regiões que apresentam fases sólidas devem ser evitadas devido ao risco de se obstruir as válvulas do lingotamento contínuo [5].

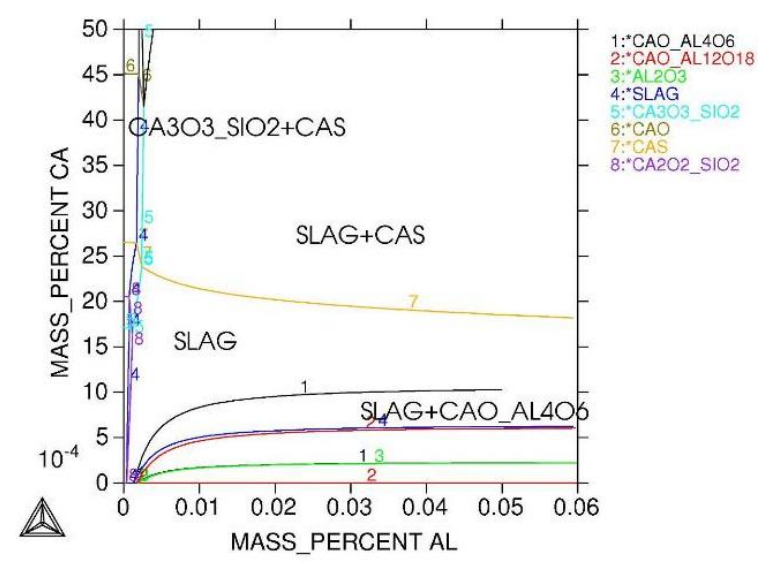

a)

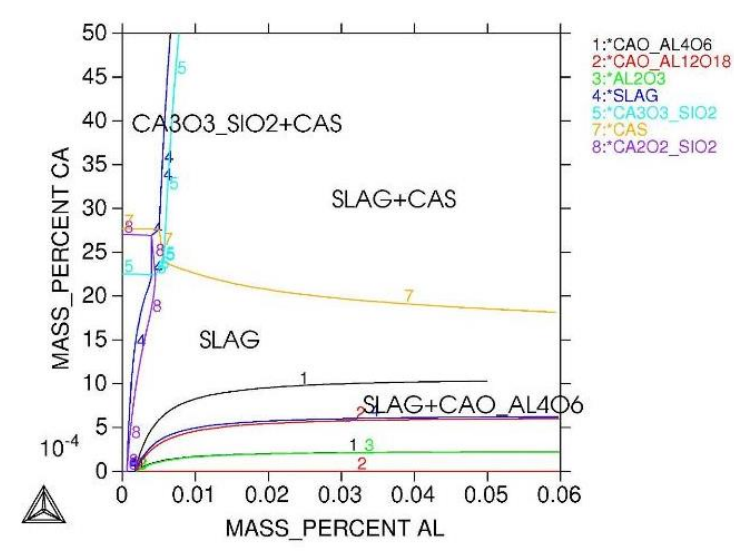

b)

Figura 3. Diagrama de fase binário alumínio vs cálcio para uma liga de aço com $0,2 \% C, 0,005 \%$ e a) $0,02 \% \mathrm{Si} \mathrm{e} \mathrm{b)} 0,20 \% \mathrm{Si}$

\footnotetext{
* Contribuição técnica ao 69ำ Congresso Anual da ABM - Internacional e ao 14ํㅡㄹ ENEMET - Encontro Nacional de Estudantes de Engenharia Metalúrgica, de Materiais e de Minas,21 a 25 de julho de 2014, São Paulo, SP, Brasil.
} 


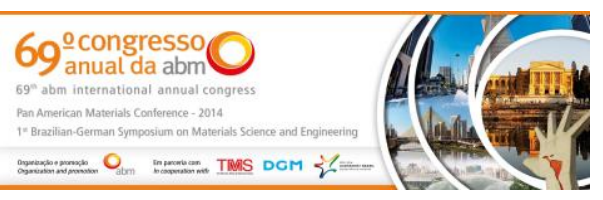

Comparando-se o resultado da Figura $3 \mathrm{a}$, para um aço com 0,02\% Si com o resultado de um aço similar, porém com teor de silício 10 vezes superior $(0,20 \%)$ observa-se uma importante diferença quanto a posição da área relativa fase sólida de silicato de cálcio. No aço de mais alto teor de silício, esta área estende-se até teores de alumínio de até $0,008 \%$ (Figura $3 b$ ).

A Figura 4 apresenta os valores calculados de teor de cálcio que devem ser objetivados para obter inclusões de fase líquida a uma temperatura $50^{\circ} \mathrm{C}$ acima da temperatura liquidus, ou seja, a quantidade de cálcio necessária para que as inclusões fiquem situadas exatamente no centro da janela de lingotabilidade. Os gráficos apresentados mostram a influência do alumínio, do enxofre, do silício e do carbono, sobre a posição da janela de lingotabilidade. É evidente que estes elementos influenciam diretamente no equilíbrio entre as fases de óxidos e sulfetos presentes no aço. O alumínio, apesar de ser o elemento de maior afinidade pelo oxigênio dentre os elementos estudados, não apresentou influência significativa na quantidade de cálcio visado, independente da quantidade dos demais elementos. Isso se deve ao fato de que a variação do alumínio na faixa de $100 \mathrm{ppm}$ a 300ppm tem pouca influência no equilíbrio do sistema. Neste caso, a quantidade de cálcio visado é praticamente constante para essa faixa de alumínio, considerando os outros elementos constantes. A variação no teor de silício de 0,02\% para 0,20\% igualmente apresenta pouca influência na quantidade de cálcio objetivado. Uma pequena diferença da ordem de 0,5ppm é observada para baixos teores de alumínio e carbono (100ppm e 0,2\% respectivamente), o que decorre em condições onde o aço tende a estar em estado de oxidação mais elevada. $\mathrm{O}$ aumento do teor de carbono de $0,2 \%$ para $0,4 \%$ influencia muito pouco na quantidade de cálcio, contudo a influência do carbono é um pouco mais pronunciada quando o teor de carbono aumenta de $0,4 \%$ para $0,6 \%$, resultado em um aumento no cálcio visado entre $0,5 \mathrm{e}$ 1 ppm. A principal alteração no teor de cálcio objetivado acontece com o aumento no teor de enxofre do aço. Os gráficos mostram que, o aumento no teor de enxofre no aço de 50ppm para 150ppm resulta na redução do teor de cálcio visado de $\sim 15 \mathrm{ppm}$ para 13ppm. Isso indica que, a presença de enxofre no banho metálico favorece a formação de sulfeto de cálcio, consequentemente o campo de fase CaS aumenta e desloca a janela de lingotabilidade para baixo, ou seja, para a região de menor teor de cálcio $[5,9,11]$.

A Figura 5 mostra o "delta cálcio", termo utilizado para definir o intervalo máximo no teor de cálcio para obter inclusões liquidas, que corresponde a toda extensão da janela de lingotabilidade para uma determinada condição. Este intervalo de cálcio representa, na prática, a dificuldade em se atingir o campo de inclusões de fase líquida após o tratamento com cálcio no refino secundário, especialmente devido à grande variação no rendimento do cálcio em fio. Como pode ser observado, o acréscimo no teor de alumínio leva a uma evidente redução no intervalo de cálcio da fase líquida. De maneira geral, cada incremento de 100ppm no teor de alumínio do aço reduz cerca de 2ppm no intervalo de cálcio. Observando-se esse aspecto, o processo de refino e lingotabilidade em aços com elevado teor de alumínio torna-se muito mais complexo que em aços com teor de alumínio moderado. A principal influência do teor de silício ocorre nas situações de baixo teor de alumínio, sendo mais realçada em aços com alto teor de carbono, onde a diferença de delta cálcio varia entre 3ppm e 4ppm para diferentes teores de silício, sendo sempre menor o intervalo de cálcio para teor de silício de $0,02 \%$. O teor de carbono da liga apresenta

\footnotetext{
* Contribuição técnica ao 69 Congresso Anual da ABM - Internacional e ao $14^{\circ}$ ENEMET - Encontro Nacional de Estudantes de Engenharia Metalúrgica, de Materiais e de Minas,21 a 25 de julho de 2014, São Paulo, SP, Brasil.
} 


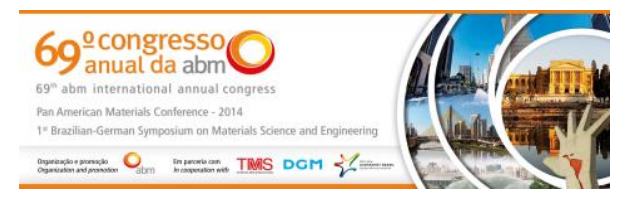

relação clara com o intervalo de cálcio. Para todas as condições, o aumento do teor de carbono provoca uma redução imediata no intervalo de cálcio necessário para obter inclusões líquidas. A redução do intervalo de cálcio é conseqüência da redução da janela de lingotabilidade, e isso provoca um aumento na probabilidade de formação de sulfetos e óxidos no estado sólido.

A quantidade de enxofre é outra forte barreira para a formação de inclusões líquidas no aço. O aumento no teor de enxofre aumenta o campo de inclusão sólida CaS e provoca um achatamento da janela de lingotabilidade. O efeito do enxofre é ainda mais pronunciado nas condições de menor oxidação do aço, ou seja, quando percentuais de carbono e alumínio se encontram em um patamar mais elevado e, consequentemente, os teores de oxigênio total devem ser menores. Na condição de $\mathrm{S}=150 \mathrm{ppm}, \mathrm{Al}=300 \mathrm{ppm}$ e $\mathrm{C}=0,4 \%$ intervalo de cálcio para ajuste da janela de lingotabilidade é de apenas 4ppm. O intervalo de cálcio é ainda menor quando o teor de carbono aumenta para $0,6 \%$, situação onde o delta cálcio reduz para apenas 2ppm.

Com os dados de cálcio visado em função das composições químicas das ligas foi então calculada uma regressão linear múltipla que correlaciona o valor de cálcio objetivado para se obter inclusões líquidas em função das quantidades dos elementos carbono, silício, enxofre e alumínio. A Figura 6 mostra os valores de cálcio visado calculados pelo software Thermocalc versus os valores de cálcio visado calculados pela regressão linear múltipla.

Observa-se que os dados apresentam em geral boa correlação, o que resultou em um $\mathrm{R}^{2}$ de $89 \%$. A regressão linear múltipla, apesar de não ser tão precisa quanto 0 software, apresenta uma vantagem operacional, uma vez que a equação pode ser inserida facilmente nos modelos metalúrgicos da aciaria e fornece uma resposta rápida da quantidade de cálcio que deve ser objetivado em relação às flutuações de composição química entre corridas no processo.

Com o objetivo de verificar o perfil das inclusões que se solidificaram no processo de lingotamento contínuo, foi retirada uma amostra transversal de tarugo de um aço com 0,20\%C/0,02\%Si/0,01\%S/0,02\%Al/0,0015\%Ca. As inclusões presentes em uma área de $23 \mathrm{~mm}^{2}$ da amostra foram avaliadas via MEV em análise semi quantitativa e representadas em diagramas ternários conectados dos sistemas $\mathrm{Al}_{2} \mathrm{O}_{3}-\mathrm{MgO}-\mathrm{CaO}$ e $\mathrm{Al}_{2} \mathrm{O}_{3}-\mathrm{CaS}-\mathrm{CaO}$ (Figura 7a).

\footnotetext{
* Contribuição técnica ao 69 Congresso Anual da ABM - Internacional e ao $14^{\circ}$ ENEMET - Encontro Nacional de Estudantes de Engenharia Metalúrgica, de Materiais e de Minas,21 a 25 de julho de 2014, São Paulo, SP, Brasil.
} 

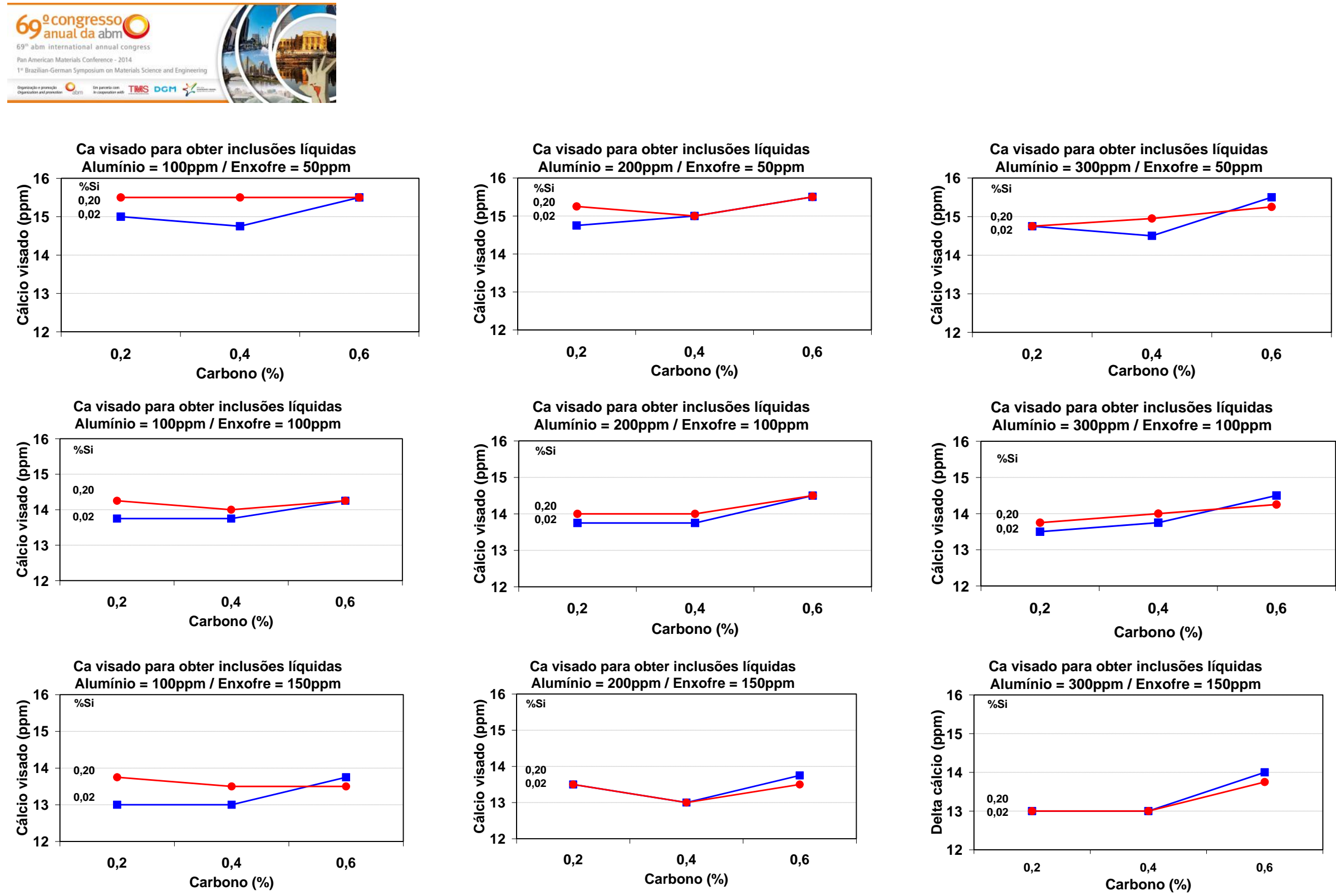

Figura 4. Teor de cálcio visado para obter inclusões líquidas. Temperatura $50^{\circ} \mathrm{C}$ acima da $\mathrm{T}$ liquidus (Tabela 1 ) e $\mathrm{Si}=0,02 \%$ e $0,20 \%$.

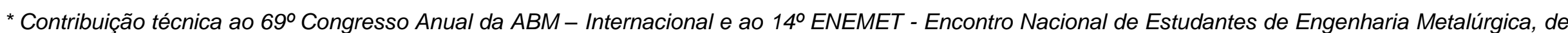
Materiais e de Minas,21 a 25 de julho de 2014, São Paulo, SP, Brasil. 

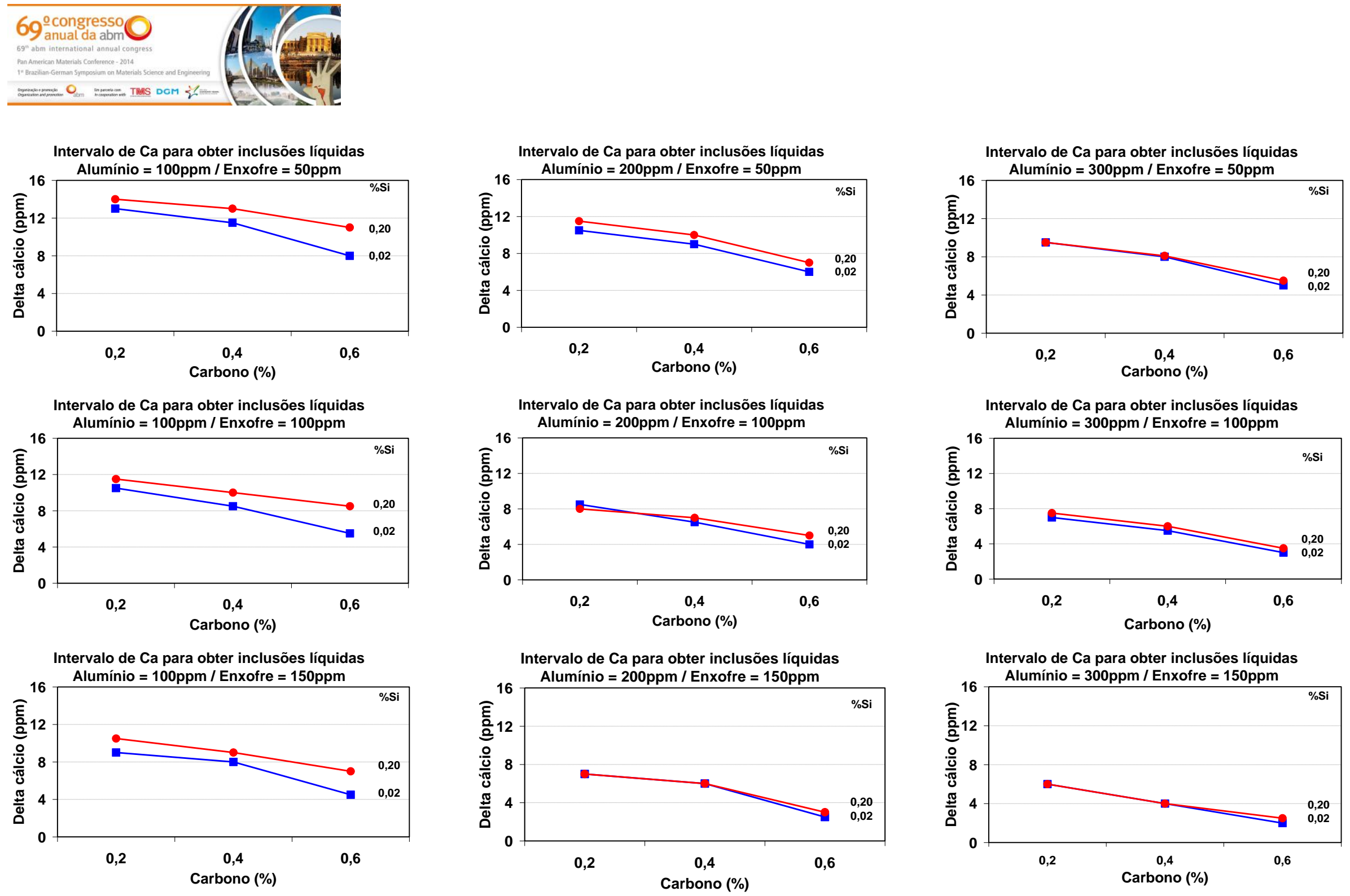

Figura 5. Intervalo no teor de cálcio para obter inclusões liquidas. Temperatura $50^{\circ} \mathrm{C}$ acima da T liquidus (Tabela 1 ) e $\mathrm{Si}=0,02 \%$ e $0,20 \%$.

* Contribuição técnica ao 69 Congresso Anual da ABM - Internacional e ao 14ํㅡㄹ ENEMET - Encontro Nacional de Estudantes de Engenharia Metalúrgica, de Materiais e de Minas,21 a 25 de julho de 2014, São Paulo, SP, Brasil. 

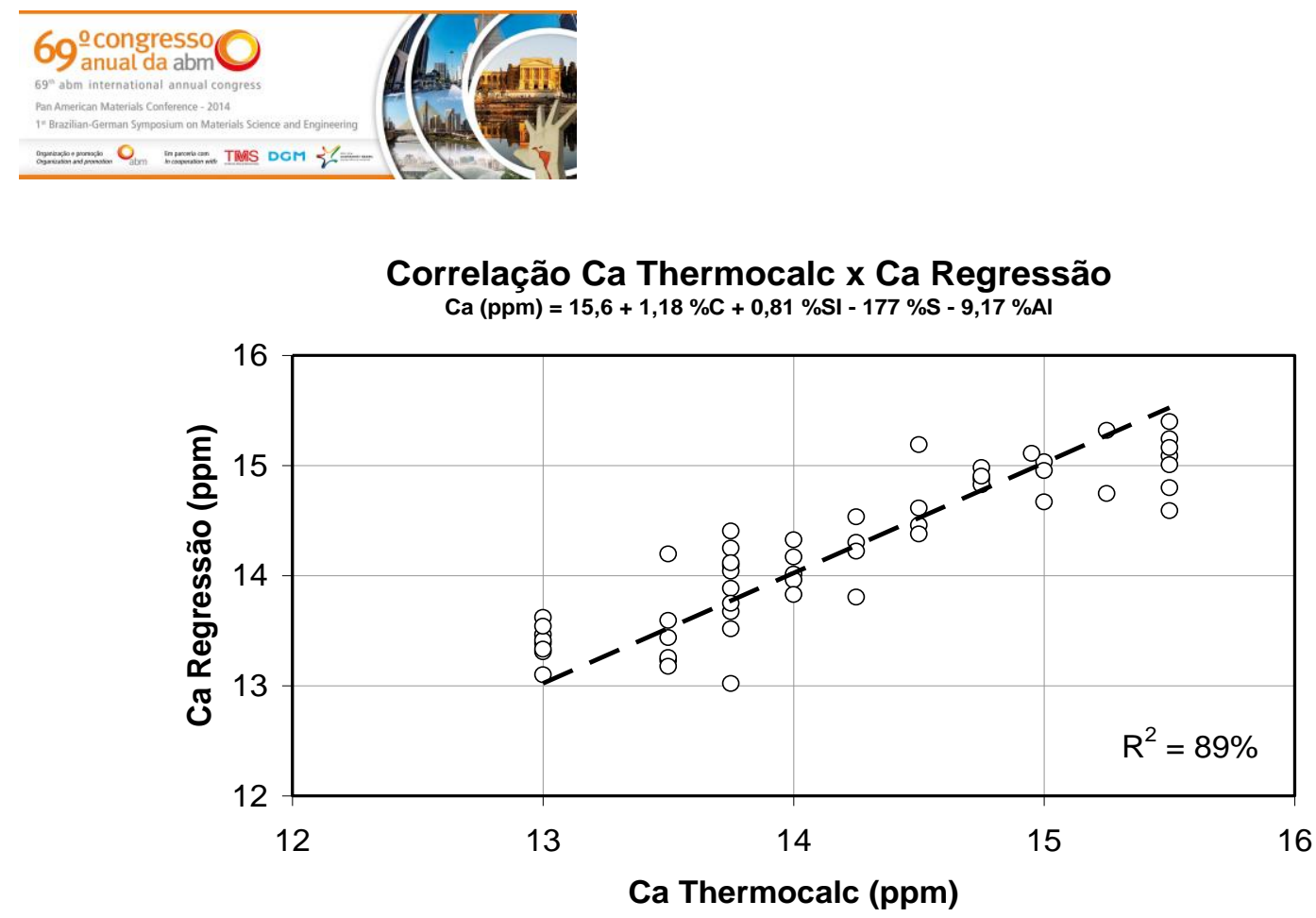

Figura 6. Valores de cálcio calculado pelo software Thermocalc e calculados a partir da regressão múltipla.

Em relação ao tamanho, $98 \%$ das inclusões apresentaram diâmetro de até $4 \mu \mathrm{m}$. O primeiro sistema $\left(\mathrm{Al}_{2} \mathrm{O}_{3}-\mathrm{MgO}-\mathrm{CaO}\right)$ tem o objetivo de ilustrar a presença da fase spinel (espinélio). Esta fase surge a partir da combinação de óxidos de alumínio formados sobretudo na desoxidação do aço e óxidos de magnésio oriundos principalmente da difusão do íon magnésio da parede refratária e da escória da panela ${ }^{10}$. A presença das fases spinel $\left(\mathrm{Al}_{2} \mathrm{O}_{3}-\mathrm{MgO}\right)$ e corundum $\left(\mathrm{Al}_{2} \mathrm{O}_{3}\right)$ é um indício de que não houve transformação completamente homogênea das inclusões do aço em aluminatos de cálcio após o tratamento com cálcio [9]. No sistema $\mathrm{Al}_{2} \mathrm{O}_{3}-\mathrm{CaS}$ $\mathrm{CaO}$ é possível identificar a presença de $\mathrm{CaS}$ em conjunto com as inclusões de aluminatos de cálcio. Na liga analisada grande parte das inclusões apresentaram fase $\mathrm{CaS}$ em sua composição. Os resultados obtidos para as inclusões desse aço estão em acordo com resultados publicados recentemente para aço baixo teor de carbono desoxidado ao alumínio (Figura 7b) [9].

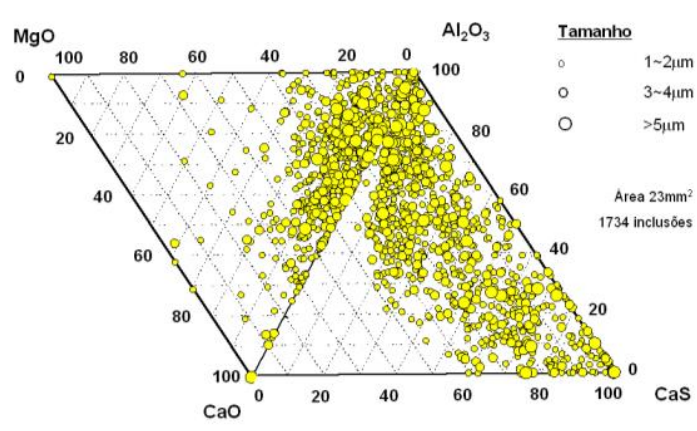

a)

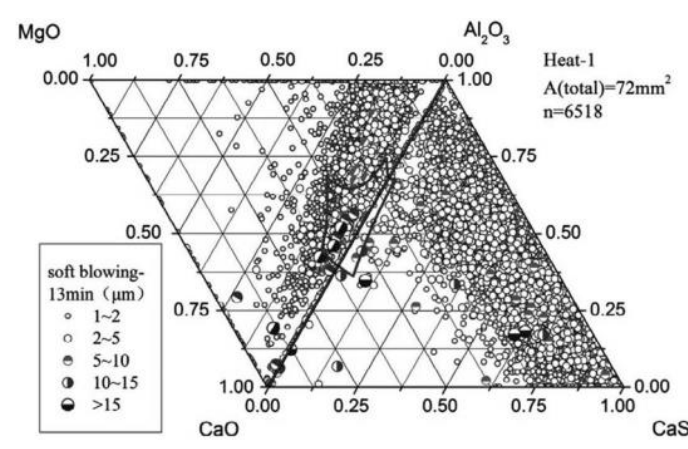

b)

Figura 7. Diagramas ternário conectados dos sistemas $\mathrm{Al}_{2} \mathrm{O}_{3}-\mathrm{MgO}-\mathrm{CaO}$ e $\mathrm{Al}_{2} \mathrm{O}_{3}-\mathrm{CaS}-\mathrm{CaO}$ da liga utilizada neste trabalho a) e resultado da literatura b) [9].

As propriedades termodinâmicas das inclusões foram calculadas utilizando 0 software CEQCSI. A Figura 8a mostra a temperatura liquidus calculada em função da composição química completa de cada inclusão.

* Contribuição técnica ao 69 Congresso Anual da ABM - Internacional e ao 14ํㅡㄹ ENEMET - Encontro Nacional de Estudantes de Engenharia Metalúrgica, de Materiais e de Minas,21 a 25 de julho de 2014, São Paulo, SP, Brasil. 

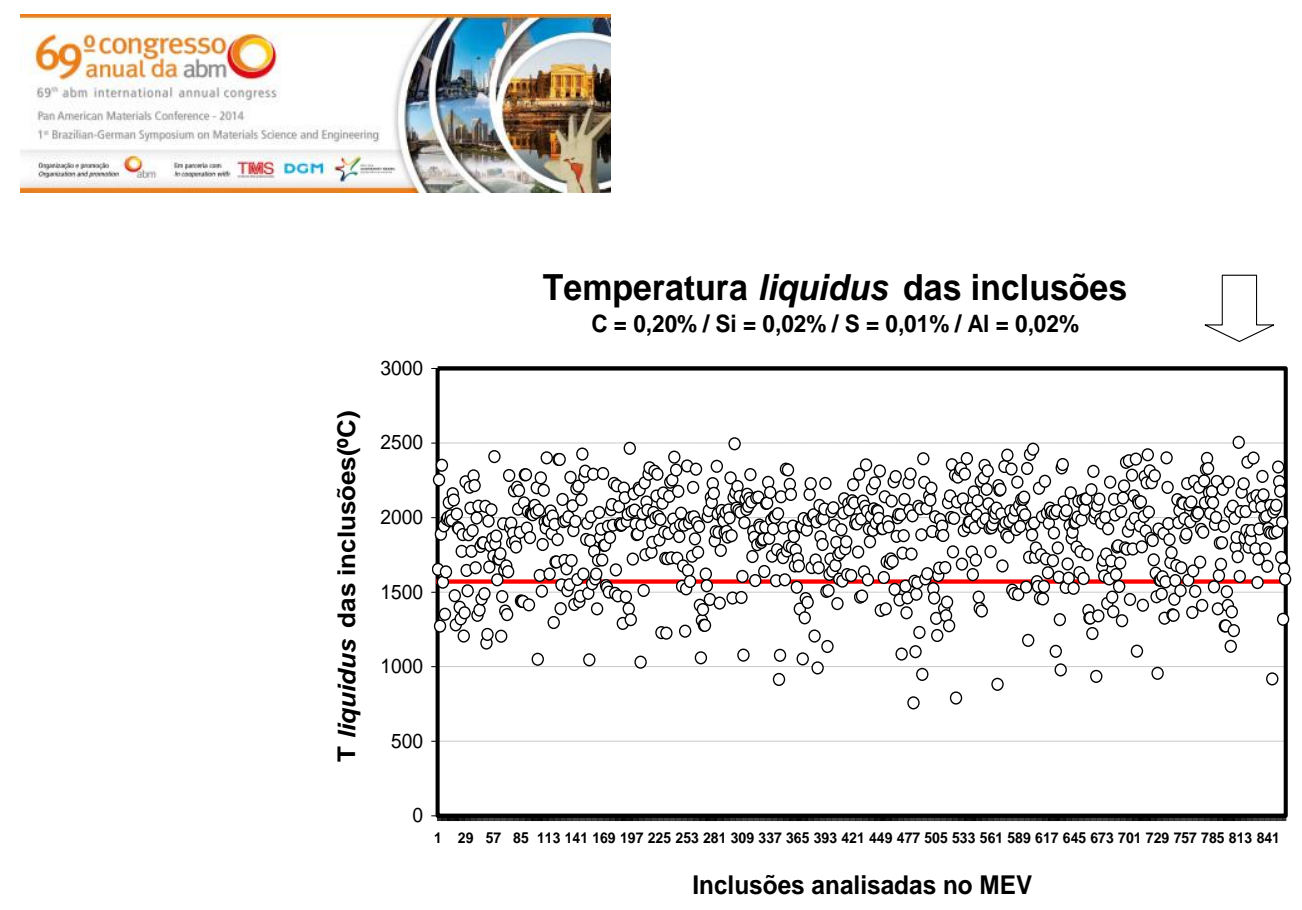

a)

Percentual de fase líquida nas inclusões

$\mathrm{C}=0,20 \% / \mathrm{Si}=0,02 \% / \mathrm{S}=0,01 \% / \mathrm{Al}=0,02 \%$

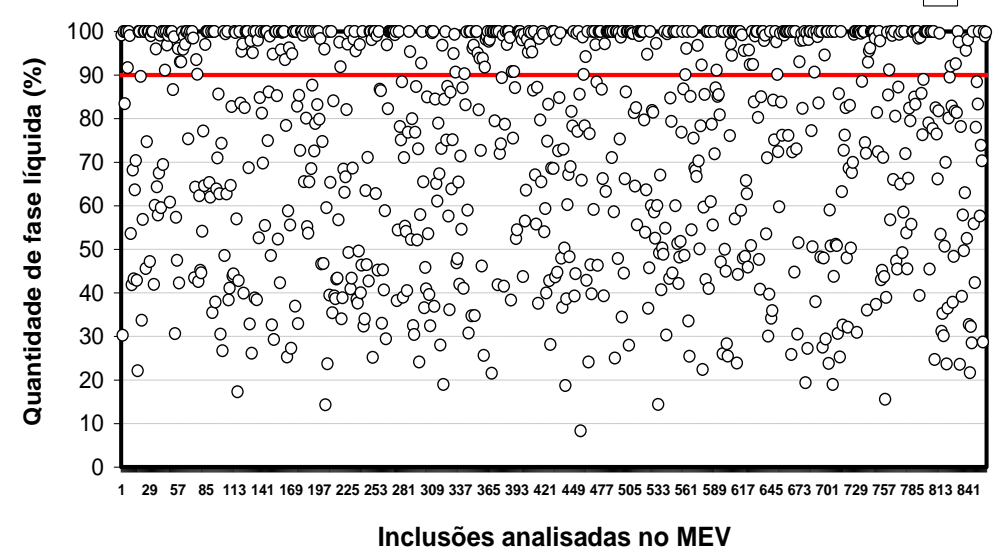

b)

Frequência de ocorrência das fases sólidas das inclusões $\mathrm{C}=0,20 \% / \mathrm{si}=0,02 \% / \mathrm{s}=0,01 \% / \mathrm{Al}=0,02 \%$

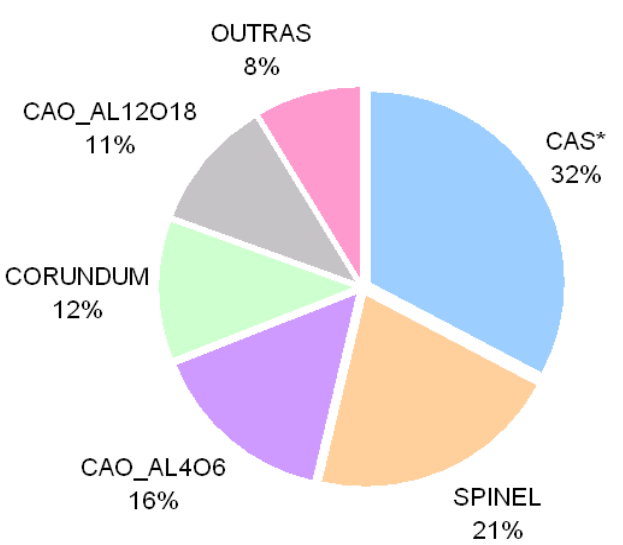

c)

Figura 8. Propriedades termodinâmicas das inclusões calculadas utilizando o software CEQCSI.

\footnotetext{
* Contribuição técnica ao $69^{\circ}$ Congresso Anual da ABM - Internacional e ao 14ํㅡㄹ ENEMET - Encontro Nacional de Estudantes de Engenharia Metalúrgica, de Materiais e de Minas,21 a 25 de julho de 2014, São Paulo, SP, Brasil.
} 


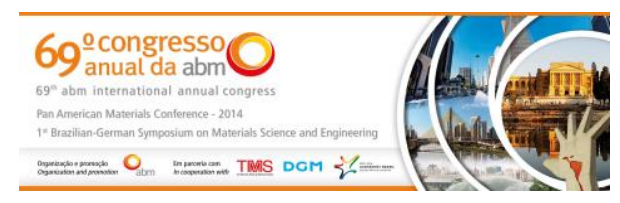

Apesar do tratamento com cálcio, nota-se que somente $20 \%$ das inclusões apresentam $\mathrm{T}$ liquidus abaixo da temperatura calculada de $50^{\circ} \mathrm{C}$ acima da $\mathrm{T}$ liquidus do aço. Isso significa que essas inclusões estarão $100 \%$ líquidas nessa temperatura Outra propriedade avaliada na Figura $8 b$ foi a quantidade de fase líquida em cada inclusão na temperatura do aço considerada. Aproximadamente $42 \%$ das inclusões apresentam, pelo menos, $90 \%$ de fase líquida na temperatura de $50^{\circ} \mathrm{C}$ acima da $\mathrm{T}$ liquidus. Neste caso, o fato de se ter inclusões parcialmente sólidas não influenciou o fluxo de aço através das válvulas do lingotamento contínuo, já que a corrida parâmetros de lingotamento considerados bons. Estas duas propriedades das inclusões são importantes, pois afetam diretamente a limpidez e lingotabilidade do aço, além das propriedades do produto final $[10,11]$. A Figura 8c mostra a frequência das fases sólidas nas inclusões que se encontram parcialmente no estado sólido. $A$ inclusão sólida de maior ocorrência é o sulfeto de cálcio (32\%). O CaS é formado predominantemente da associação do cálcio adicionado com o enxofre dissolvido no aço. As inclusões de sulfeto formadas a temperaturas elevadas normalmente precipitam ao redor dos aluminatos formados previamente [9]. Essa característica é bastante interessante do ponto de vista da aplicação do produto, pois favorece a morfologia esférica das inclusões que melhora os resultados relativos à formação de microtrincas e a resistência a fadiga [7].

Por outro lado, a precipitação de CaS em excesso pode prejudicar o processo de lingotamento contínuo uma vez que reduz a janela de lingotabilidade do aço, e por isso deve ser evitado [6]. Outras fases sólidas de maior freqüência nas inclusões são spinel, aluminatos de cálcio e corundum. A presença dessas inclusões é um indicativo que a modificação das inclusões ocorreu de maneira incompleta. Concluise que, apesar de efetivo, o tratamento com cálcio apresenta certo grau de heterogeneidade. Contudo, ainda é bastante funcional na prática, pois permite o lingotamento contínuo de longos seqüenciais de aços desoxidados ao alumínio e com a garantia de qualidade assegurada dos produtos produzidos por esse processo.

\section{CONCLUSÕES}

Através deste trabalho, as seguintes conclusões foram obtidas:

1. A quantidade de cálcio visada e o intervalo de cálcio da janela de lingotabilidade foram estimados através de cálculos de termodinâmica computacional. A quantidade de cálcio adicionada é de grande importância, pois permite a modificação das inclusões e a sustentabilidade do processo de lingotamento contínuo.

2. Nas condições avaliadas, um aumento nos teores de carbono, alumínio e enxofre reduzem a janela de lingotabilidade. Por outro lado, um aumento no teor de silício aumenta a janela de lingotabilidade.

3. Foi desenvolvida uma equação de regressão múltipla linear com boa correlação $\mathrm{R}^{2}$, capaz de prever de maneira mais rápida que o cálculo termodinâmico as quantidades de cálcio necessárias, em função dos teores de alumínio, silício, carbono e enxofre.

4. Os diagramas ternário produzidos a partir da análise semi-quantitativa em MEV mostrou a presença de inclusões do tipo spinel, corundum e

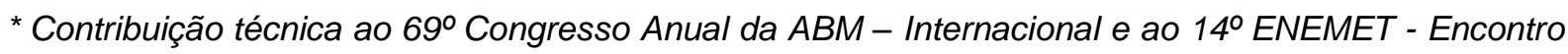
Nacional de Estudantes de Engenharia Metalúrgica, de Materiais e de Minas,21 a 25 de julho de 2014, São Paulo, SP, Brasil.
} 


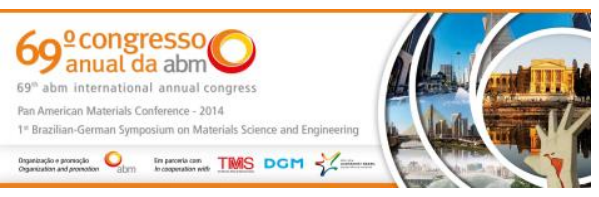

CaS/aluminatos. As fases spinel e corundum indicam uma transformação heterogênea das inclusões após o tratamento com cálcio.

5. $\mathrm{Na}$ análise das inclusões de tarugo de um aço com 0,20\%C/0,02\%Si/0,01\%S/0,02\%Al/0,0015\%Ca, 20\% das inclusões apresentam temperatura liquidus abaixo da temperatura de lingotamento e $42 \%$ das inclusões apresentam mais de $90 \%$ de fase líquida. Apesar de ter característica heterogênea, o tratamento com cálcio permite o lingotamento contínuo de aços desoxidados ao alumínio.

\section{REFERÊNCIAS}

1 Van EM, Guo M, Zinngrebe E, Blanpain B, Jung lj. Evolution of Non-Metallic Inclusions in Secondary Steelmaking: Learning from Inclusion Size Distributions. ISIJ International, 2013; 53(11): 1974-1982.

2 Choudhary Sk, Chandra S. Castability of Mn-Si Killed Low Carbon Wire Rod Steel. ISIJ International, 2007; 47(1): 190-192.

3 Yang W, Zhang L, Wang X, Ren Y, Liu X, Shan Q. Characteristics of Inclusions in Low Carbon Al-Killed Steel during Ladle Furnace Refining and Calcium Treatment. ISIJ International, 2013; 53 (8): 1401-1410.

4 Silva AVC. Aplicações da termodinâmica computacional aplicada a aciaria. Curso integrado ao XXXVII Seminário de Aciaria Internacional da ABM. Porto Alegre, Brasil, Maio, 2006.

5 Magalhaes $\mathrm{H}$, Silva CA, Silva AVC. Melhoria da limpidez do aço SAE 1045 desoxidado ao alumínio com aplicação na indústria automobilística, utilizando termodinâmica computacional, REDEMAT/UFOP, Ouro Preto, Fevereiro, 2010.

6 Costa Neto JG, Lino RE, Marins AMF, Marchi LA, Ferreira HC. Termodinâmica Computacional: Aplicações Práticas na Fabricação do Aço. 67 Congresso da ABM Internacional - 31 de julho a 3 de agosto de 2012 - Rio de Janeiro - RJ - Brasil.

7 Jiang M, Wang X, Chen B, Wang W. Laboratory Study on Evolution Mechanisms of Nonmetallic Inclusions in High Strength Alloyed Steel Refined by High Basicity Slag. ISIJ International, 2010; 50(1): 95-104.

8 Turkdogan ET. Fundamentals of Steelmaking, London: The Institute of Materials, 1996. Chapter 9.

9 Yang W, Zhang L, Wang X, Ren Y, Liu X, Shan Q. Characteristics of Inclusions in Low Carbon Al-Killed Steel during Ladle Furnace Refining and Calcium Treatment. ISIJ International, 2013; 53 (8): 1401-1410.

10 Park Jh, Todoroki H. Control of MgO-Al2O3 Spinel Inclusions in Stainless Steels. ISIJ International, 2010; 50 (10): 1333-1346.

11 Choudhary Sk, Ghosh A. Thermodynamic Evaluation of Formation of Oxide-Sulfide Duplex Inclusions in Steel. ISIJ International, 2008; 48 (11): 1552-1559.

12 Lino RE, Marins AMF, Penna LV, Costa Neto JG, Caldeira JHP, Silva AvC. Determinação de temperatura liquidus de aços. 68을 Congresso da ABM - Internacional 30 de julho a 2 de agosto de 2013 - Belo Horizonte - MG - Brasil.

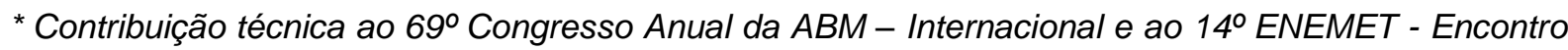
Nacional de Estudantes de Engenharia Metalúrgica, de Materiais e de Minas,21 a 25 de julho de 2014, São Paulo, SP, Brasil.
} 